\title{
The orphan nuclear receptor Nur77 regulates hepatic cholesterol metabolism through the suppression of LDLR and HMGCR expression
}

\author{
PENG ZHANG, YANWEI HU, JUNYAO YANG, LEI ZHENG and QIAN WANG \\ Department of Laboratory Medicine, Nanfang Hospital, Southern Medical University, \\ Guangzhou, Guangdong 510515, P.R. China
}

Received December 28, 2011; Accepted March 26, 2012

DOI: $10.3892 / \mathrm{mmr} .2012 .850$

\begin{abstract}
Disorders in cholesterol metabolism are critical in development of atherosclerosis and are related to acute myocardial infarction (AMI). The liver is one of the most important organs that balances cholesterol metabolism. In order to investigate whether Nur77 is capable of regulating cholesterol metabolism in HepG2 cells and to demonstrate the underlying mechanism, the downregulation and upregulation of Nur77 expression in HepG2 cells was achieved by the transfection of siRNA specific to Nur77 and the transfection of the recombinant plasmid, pcDNA3.1-Nur77, respectively. Following the downregulation and upregulation of Nur77 expression, changes in the total cholesterol (TCHO) levels in HepG2 cells were observed based on lipid overloading. Thereafter, changes in a series of key gene expressions related to hepatic cholesterol metabolism were measured at the mRNA and protein levels by RT-PCR and western blot analysis, respectively. The TCHO levels in the HepG2 cells were found to increase following the downregulation of Nur77 expression and to decrease following the upregulation of Nur77 expression; these results were confirmed by oil red $\mathrm{O}$ staining of the cells. As for the hepatic cholesterol metabolism genes, low-density lipoprotein receptor (LDLR) and HMGCoA reductase (HMGCR) levels increased following the downregulation of Nur77 expression and decreased following the upregulation of Nur77 expression. However, liver X receptor $\alpha(\mathrm{LXR} \alpha)$ expression did not change markedly along with that of Nur77. According to these findings, we conclude that Nur77 is capable of reducing hepatic
\end{abstract}

Correspondence to: Professor Qian Wang, Department of Laboratory Medicine, Nanfang Hospital, Southern Medical University, Guangzhou North Street No. 1838, Guangzhou, Guangdong 510515, P.R. China

E-mail: nfyyzp@126.com

Key words: Nur77, HepG2 cells, cholesterol metabolism, lowdensity lipoprotein receptor, HMGCoA reductase cholesterol based on lipid overloading, and that this may be due to the decrease in LDLR and HMGCR levels.

\section{Introduction}

To date, an increasing number of individuals worldwide are experiencing cholesterol metabolism disorders. These disorders are one of the key factors of atherosclerosis development and are related to a number of serious diseases, such as acute myocardial infarction (AMI) and stroke $(1,2)$. The liver is the organ that plays a role in reverse cholesterol transport and is responsible for the excretion of cholesterol. Abnormal lipid profiles are often observed in patients with liver diseases (3). Therefore, much of the research has been focused on the hepatic cell when investigating cholesterol metabolism disorders.

Nur77 is also known as nuclear receptor subfamily 4 group A member 1 (NR4A1) and nerve growth factor I-B (NGFI-B), and was first discovered by Milbrandt in 1988 in the pheochromocytoma cell line, $\mathrm{PC12}$, after being induced by the nerve growth factor (4). Nur77 belongs to the NR4A family of nuclear receptors, which also includes Nurr1 (NR4A2) and Nor1 (NR4A3). As with other nuclear receptors, it consists of an N-terminal activating-function-1 domain, a central DNA-binding domain, and a C-terminal ligand-binding domain (LBD) (5). However, the LBD of Nur77 is not typical and lacks the classical ligandbinding pocket (6). Therefore, it has been demonstrated that Nur77 is regulated by differential gene expression and posttranslational modification rather than ligand-binding $(7,8)$. It has been reported that Nur77 plays a role in the regulation of a number of biological process including apoptosis, inflammation and glucose metabolism (9-16). It has also been reported that Nur77 is involved in hepatic lipid metabolism in mice, and can lower hepatic triglyceride levels through the suppression of SREBP1c activity (17). However, there is no significant evidence on whether Nur77 is capable of regulating the hepatic cholesterol metabolism, particularly in humans.

In this study, we demonstrate for the first time that Nur77 reduces hepatic cholesterol based on lipid overloading, and the underlying mechanism may lie in the decrease in the levels of the low density lipoprotein receptor (LDLR) and HMGCoA reductase (HMGCR). 


\section{Materials and methods}

Cell culture. The HepG2 cells were purchased from the American Type Culture Collection (ATCC). The cells were maintained in Dulbecco's modified Eagle's medium (Gibco Invitrogen Life technologies, Shanghai, China) supplemented with $10 \%$ heat-inactivated fetal bovine serum (Gibco Invitrogen Life technologies).

RNA interference. An siRNA segment specific for Nur77 was synthesized by Ribobio Co. Ltd. (Guangzhou, China). The sequence is shown in Table I. The transfection process was carried out using Lipofectamine 2000 transfection reagent (Gibco Invitrogen Life technologies) according to the manufacturer's instructions. In order to achieve the optimal downregulation of Nur77 expression, the duration of the transfection process and the ratio of the siRNA fragment and Lipofectamine 2000 were optimized, respectively.

Construction and transfection of the recombinant plasmid. The PIRES2-EGFP vector and PCR-XL-TOPO vector (containing Nur77 which was assembled by the chemically synthesized oligos through PCR) were purchased from Invitrogen Life technologies. Segments of EcoRI-Nur77 and IRES-EGFP-XhoI were amplified using the template of the PCR-XL-TOPO and PIRES2-EGFP vectors, respectively. Gel electrophoresis was carried out and the 1,800 and 1,300 bp bands were retrieved, respectively. EcoRI-NR4A1-IRES-EGFP-XhoI was then jointed by the two segments mentioned above using overlap PCR. Gel electrophoresis was carried out again and the 3,100 bp band was retrieved. The retrieved fragment was then double enzyme digested by EcoRI/XhoI (Takara Biotechnology Co., Ltd., Dalian, China), and was linked to the pcDNA3.1(+)vector (Takara Biotechnology Co., Ltd.). It was then transformed into the competent cells, DH5a, (Takara Biotechnology Co., Ltd.), for further amplification and use. The recombinant plasmid was verified by sequencing and named as pcDNA3.1-Nur77.

The plasmid transfection process was carried out using Lipofectamine 2000 transfection reagent according to the manufacturer's instructions. The ratio of plasmid and Lipofectamine 2000 was optimized to $1: 2.5(\mu \mathrm{g}: \mu \mathrm{l})$.

Quantification of total cholesterol (TCHO) in HepG2 cells. In order to achieve the lipid overload model of the HepG2 cell line, a mixture of oleate/palmitate (at a ratio of 2:1) was added into the regular DMEM culture $6 \mathrm{~h}$ following the transfection of the siRNA segment and recombinant plasmid, respectively. The final concentration of the mixture was diluted to $0.5 \mathrm{nM}$ as described previously (18). A total of $48 \mathrm{~h}$ after lipid overloading, different groups of the cells were dissociated with RIPA buffer (Biocolors Biotechnologies, Shanghai, China). The profiles of TCHO and total protein (TP) levels were quantified by the automated biochemistry analyzer, AU5400 (Beckman Coulter Ltd., USA), using the kits of Ausbio Laboratories Co., Ltd. (Yantai, China) and Beckman Coulter Co., Ltd., respectively. The TCHO levels of the different groups were corrected for the level of TP.

Oil red $O$ staining. As for the $\mathrm{HepG} 2$ cell staining, the cells were rinsed 3 times with PBS and fixed in 10\% formalin for
$30 \mathrm{~min}$. They were briefly washed with running tap water for 2 min and stained with freshly prepared oil red $\mathrm{O}$ working solution for $15 \mathrm{~min}$. The cells were then rinsed with $60 \%$ isopropanol and distilled water, then the lipids in the cells were observed to be clearly stained red.

RNA extraction, $c D N A$ synthesis and RT-PCR analysis. RNA was extracted from the cells using RNAiso plus (Takara Biotechnology Co., Ltd.) according to the manufacturer's instructions. Thereafter, the cDNA was synthesized from $1 \mu \mathrm{g}$ total RNA using a PrimeScript RT reagent kit (Takara Biotechnology Co. Ltd.). Quantitative RT-PCR was performed using SYBR Premix Ex Taq (Takara Biotechnology Co. Ltd.) in the ABI 7500 fast RT-PCR system with gene-specific primers. Primer sequences were designed and are shown in Table II. All expression levels were corrected to the expression of the housekeeping gene, GAPDH.

Western blot analysis. Protein was extracted from the cells using RIPA buffer (Biocolors Biotechnologies). The protein concentration was quantified using the BCA protein assay kit (KeyGen Biotechnologies, Nanjing, China). A sample of $50 \mu \mathrm{g}$ TP was used to carry out the western blot analysis with the PowerPac Basic Electrophoresis system (BioRad, Singapore). Specific antibodies were synthesized by Abcam Ltd. (Hong Kong, China). All expression levels were corrected to the expression of the housekeeping protein, $\beta$-actin.

Statistical analysis. All data were retrieved from 3 independent experiments, and shown as the means \pm standard deviation (SD). The paired t-test was used to calculate statistical significance using SPSS13.0 software. $\mathrm{P}<0.05$ was considered to indicate a statistically significant difference.

\section{Results}

Downregulation of Nur77 expression by RNA interference. Following the optimization of transfection time and siRNA dose, the optimal downregulation of Nur77 expression was achieved following transfection at a final siRNA concentration of $100 \mathrm{nM}$ for $36 \mathrm{~h}$. According to the RT-PCR analysis, the optimal downregulation (approximately 10\%) was achieved $36 \mathrm{~h}$ following the transfection using the siRNA dose of $100 \mathrm{nM}$. Furthermore, the downregulatory effect was confirmed by western blot analysis $48 \mathrm{~h}$ following transfection of the siRNA segment at a final concentration of $100 \mathrm{nM}$. The results were in agreement with those of the RT-PCR analysis (Fig. 1).

Identification of the recombinant plasmid and upregulation of Nur77 expression. The inserted gene 'EcoRI-NR4A1-IRESEGFP-XhoI' was amplified by overlap PCR as described in Materials and methods. It was then linked into the pcDNA3.1(+) vector and amplified by DH5 $\alpha$. The recombinant plasmid (pCDNA3.1-Nur77) was then double enzyme digested (EcoRI/XhoI), and the result of the gel electrophoresis is shown in Fig. 2. Bands of 3.1 and $5.4 \mathrm{~kb}$ may be observed clearly in Fig. 2. The total sequencing data of the inserted gene was combined and the total length was also $3.1 \mathrm{~kb}$, which implied that the construction of the recombinant plasmid was successful. 
Table I. siRNA fragments synthesized for Nur77.

Target sequence

Synthesized sequence

CGGCTACACAGGAGAGTTT

\section{5'-CGGCUACACAGGAGAGUUU dTdT-3' \\ 3'-dTdT GCCGAUGUGUCCUCUCAAA-5'}

Table II. Primers for RT-PCR amplification of the genes.

Name Sequence (5'-3')

$\begin{array}{ll}\text { GAPDH-forward } & \text { GCACCGTCAAGGCTGAGAAC } \\ \text { GAPDH-reverse } & \text { TGGTGAAGACGCCAGTGGA } \\ \text { Nur77-forward } & \text { CCAGCACTGCCAAACTGGACTA } \\ \text { Nur77-reverse } & \text { CTCAGCAAAGCCAGGGATCTTC } \\ \text { LDLR-forward } & \text { GGCAGTGTGACCGGGAATATG } \\ \text { LDLR-reverse } & \text { TTCGCCGCTGTGACACTTG } \\ \text { HMGCR-forward } & \text { GCCTGGCTCGAAACATCTGAA } \\ \text { HMGCR-reverse } & \text { CTGACCTGGACTGGAAACGGATA } \\ \text { LXR } \alpha \text {-forward } & \text { TCTGGAGACATCTCGGAGGTACAAC } \\ \text { LXR } \alpha-\text {-reverse } & \text { AGCAAGGCAAACTCGGCATC }\end{array}$

LDLR, low density lipoprotein receptor; HMGCR, HMGCoA reductase; LXR $\alpha$, liver X receptor $\alpha$.

A total of $48 \mathrm{~h}$ following transfection of the recombinant plasmid, the expression level of Nur77 mRNA increased markedly (approximately 500-fold higher than the control) according to the RT-PCR analysis. Similarly, the expression level of the Nur77 protein markedly increased $48 \mathrm{~h}$ following the transfection of the plasmid, pCDNA3.1-Nur77, compared with the control (pcDNA3.1-mock), which can be observed clearly in the western blot analysis results (Fig. 3).

Nur77 reduces TCHO levels in Hep G2 cells. Five groups of cells were included in this experiment. No transfection was carried out in the blank group. siRNA-NC and siRNA-Nur77 were the downregulated groups. They were transfected with the negative control of the siRNA segment and siRNA specific for Nur77, respectively. pcDNA3.1-mock and pcDNA3.1-Nur77 were the upregulated groups. They were transfected with the empty pcDNA3.1 plasmid and the recombinant plasmid pcDNA3.1, respectively. The regular DMEM culture was changed into the lipid overload culture mentioned previously (15), $6 \mathrm{~h}$ after each transfection. A total of $48 \mathrm{~h}$ later, the TCHO levels in HepG2 cells were quantified after correction to the level of TP. The TCHO levels in the HepG2 cells showed an 8.8\% increase following the downregulation of Nur77 expression $(0.124 \pm 0.017 \mathrm{mmol} / \mathrm{gTP}$ vs. $0.114 \pm 0.019 \mathrm{mmol} / \mathrm{gTP})$. On the other hand, TCHO levels showed a $14.2 \%$ decrease following the upregulation of Nur77 expression $(0.091 \pm 0.017 \mathrm{mmol} /$ gTP vs. $0.106 \pm 0.01 \mathrm{mmol} / \mathrm{gTP})$. The changes were statistically significant (Table III).

Oil red $\mathrm{O}$ staining was performed in the five groups after the same number of lipid overload hours. The results are in agreement with the TCHO quantification results, as is shown in Fig. 4.

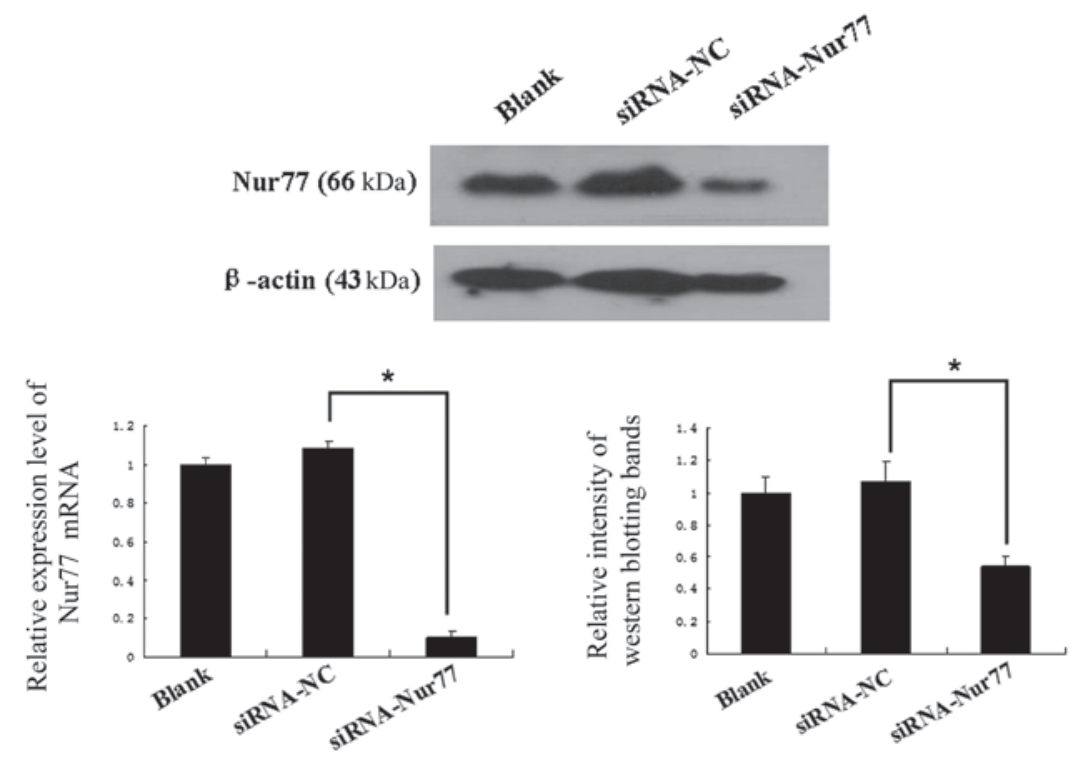

Figure 1. Downregulation of Nur77 expression by of RNA interference. The siRNA concentration of $100 \mathrm{nM}$ was used to achieve the downregulation of Nur77 expression. The relative expression level of Nur77 mRNA dereased to approximately $10 \% 36 \mathrm{~h}$ following the transfection of the siRNA segment according to the RT-PCR analysis. Compared with the negative control (siRNA-NC), the difference was statistically significant ("P<0.01). This downregulatory effect was confirmed by western blot analysis $48 \mathrm{~h}$ following the transfection of the siRNA segment $\left({ }^{*} \mathrm{P}<0.01\right)$. 
Table III. TCHO levels in HepG2 cells following the downregulation and upregulation of Nur77 expression.

\begin{tabular}{|c|c|c|c|c|c|}
\hline & Blank & siRNA-NC & siRNA-Nur77 & pcDNA3.1-mock & pcDNA3.1-Nur77 \\
\hline $\begin{array}{l}\text { TCHO } \\
(\mathrm{mmol} / \mathrm{l})\end{array}$ & $0.933 \pm 0.132$ & $0.507 \pm 0.075$ & $0.673 \pm 0.080$ & $0.503 \pm 0.064$ & $0.393 \pm 0.038$ \\
\hline $\begin{array}{l}\mathrm{TP} \\
(\mathrm{g} / \mathrm{l})\end{array}$ & $8.567 \pm 0.058$ & $4.467 \pm 0.252$ & $4.633 \pm 0.058$ & $4.800 \pm 0.265$ & $4.900 \pm 0.200$ \\
\hline $\begin{array}{l}\mathrm{TCHO} / \mathrm{TP} \\
(\mathrm{mmol} / \mathrm{gTP}) \\
\mathrm{t} \\
\mathrm{P} \text { value }\end{array}$ & $0.109 \pm 0.015$ & $0.114 \pm 0.019$ & $\begin{array}{c}0.145 \pm 0.017 \\
-15.522^{\mathrm{a}} ;-14.923^{\mathrm{b}} \\
0.004^{\mathrm{a}} ; 0.004^{\mathrm{b}}\end{array}$ & $0.106 \pm 0.018$ & $\begin{array}{c}0.081 \pm 0.011 \\
11.625^{\mathrm{a}} ; 5.274^{\mathrm{c}} \\
0.007^{\mathrm{a}} ; 0.034^{\mathrm{c}}\end{array}$ \\
\hline
\end{tabular}

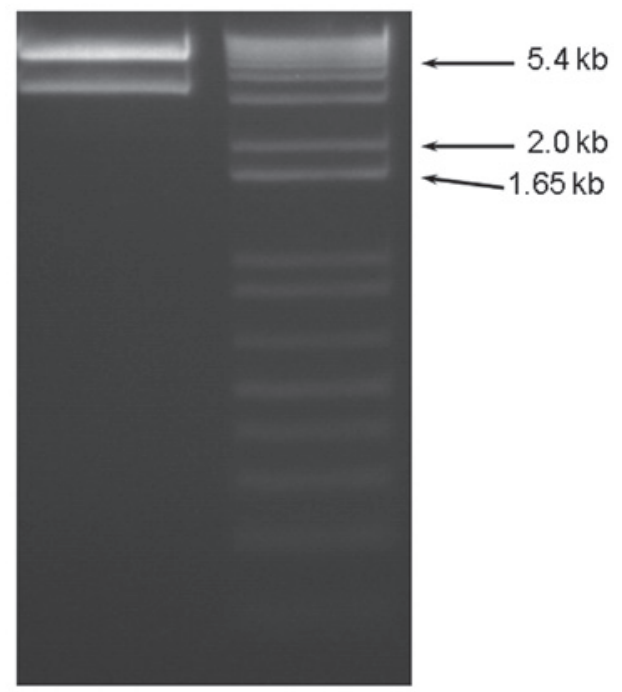

Figure 2. Electrophoresis results of the double enzyme digested (EcoRI/XhoI) plasmid. The EcoRI and XhoI enzymes were used to carry out the digestion of the recombinant plasmid according to the pcDNA3.1(+) manual and the design of the overlap PCR. After carrying out electrophoresis of the digestion products, bands of 3.1 and $5.4 \mathrm{~kb}$ can be observed clearly, which represent the inserted Nur77 and the rest of the pcDNA3.1(+) plasmid, respectively.

Nur77 modulates hepatic cholesterol metabolism gene expression. In order to investigate the mechanism underlying the reduction in hepatic TCHO levels in response to the upregulation of Nur77 expression, we performed mRNA expression analysis of a series of genes that are critical in hepatic cholesterol metabolism, including LDLR, HMGCR and LXR $\alpha$. According to the downregulation and upregulation in the expression of Nur77, RNA was extracted from the cells $36 \mathrm{~h}$ following siRNA transfection, and $48 \mathrm{~h}$ following the transfection of the recombinant plasmid, respectively. RT-PCR analysis was then performed and the mRNA expression was corrected to that of the housekeeping gene, GAPDH. LDLR and HMGCR levels were found to increase following the downregulation of Nur77 expression and to decrease following the upregulation of Nur77 expression. However, the expression of LXR $\alpha$ did not change markedly along with that of Nur77. All these trends were confirmed by western blot analysis performed $48 \mathrm{~h}$ following the downregulation and upregulation of Nur77 expression, which may be observed clearly in Fig. 5.

\section{Discussion}

The liver is one of the most important organs that plays a role in cholesterol metabolism and helps to maintain the balance of serum lipid profiles. Nur77 has been reported to play a role in the regulation of lipid metabolism. The hepatic expression of Nur77 via adenoviral overexpression vectors in mice has been shown to modulate the plasma lipid profiles, and reduce hepatic triglyceride content $(17,19,20)$. It has also been reported that Nur77 null mice on a high-fat diet exhibit increased hepatic steatosis (15). However, all these studies have focused on Nur77 and the regulation of hepatic triglyceride metabolism, and it is not clear whether Nur77 plays a role in the regulation of hepatic cholesterol metabolism.

Using the siRNA oligonucleotides and the recombinant plasmid specific for Nur77, we successfully achieved the downregulation and upregulation of Nur77 expression in HepG2 cells. Based on lipid overloading, the TCHO level in HepG2 cells was found to increase following the downregulation of Nur77 expression and to decrease following the upregulation of Nur77 expression. This phenomenon confirms that Nur77 does play a role in the regulation of hepatic cholesterol metabolism.

We then investigated the mechanism underlying this phenomenon by examining a series of genes which are critical in hepatic cholesterol metabolism. LDLR and HMGCR levels were found to increase following the downregulation of Nur77 expression and to decrease following the upregulation of Nur77 expression; yet LXR $\alpha$ expression did not change markedly along with that of Nur77. LDLR plays an important role in the clearance of plasma-activated $\alpha 2$-macroglobulin and apolipoprotein E-enriched lipoproteins (21). It is highly expressed in the hepatic cell and is responsible for the absorp- 


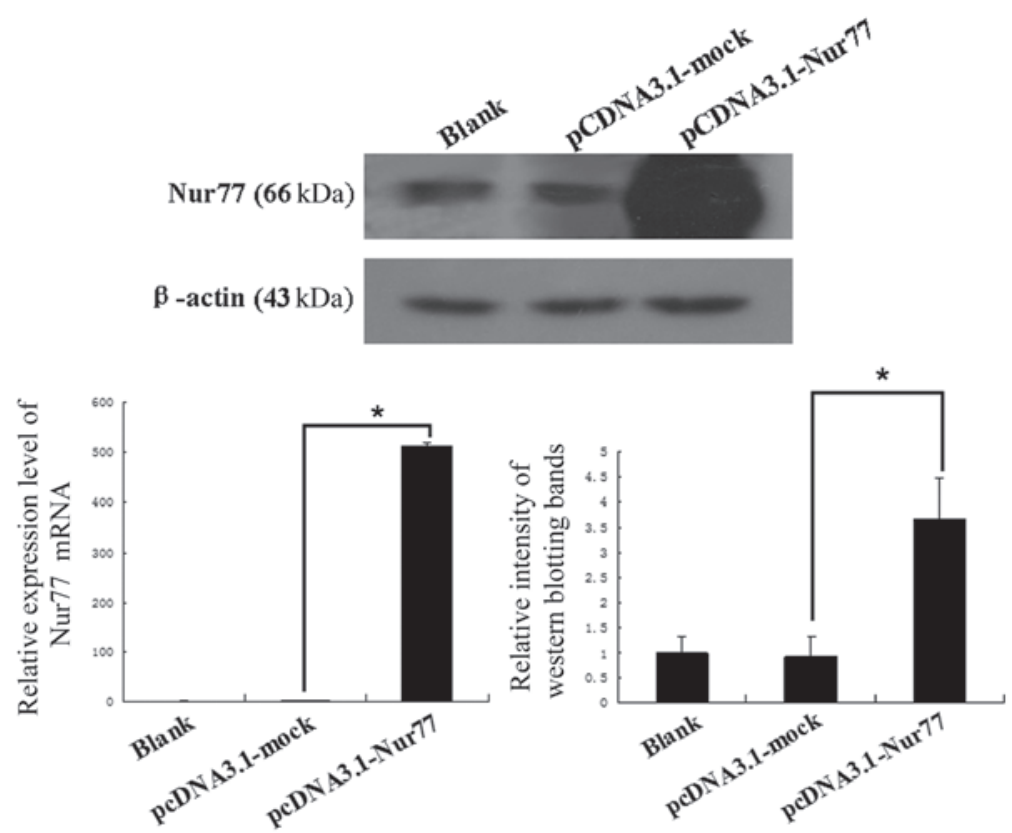

Figure 3. Upregulation of Nur77 expression using the recombinant plasmid. According to the RT-PCR analysis, the relative expression level of Nur77 mRNA increased to approximately 500-fold higher than that of the control (pcDNA3.1-mock) $48 \mathrm{~h}$ following the transfection of the plasmid, pCDNA3.1-Nur77. The difference between the 2 groups was statistically significant $(" \mathrm{P}<0.01)$. The relative expression level of Nur77 mRNA in the pcDNA3.1-mock group was very low compared with that of the pcDNA3.1-Nur77 group; the bar can barely be observed, which implied that the upregulatory effect was marked. This upregulatory effect was confirmed by western blot analysis $48 \mathrm{~h}$ following the transfection of the recombinant plasmid ( $\mathrm{P}<0.01)$.

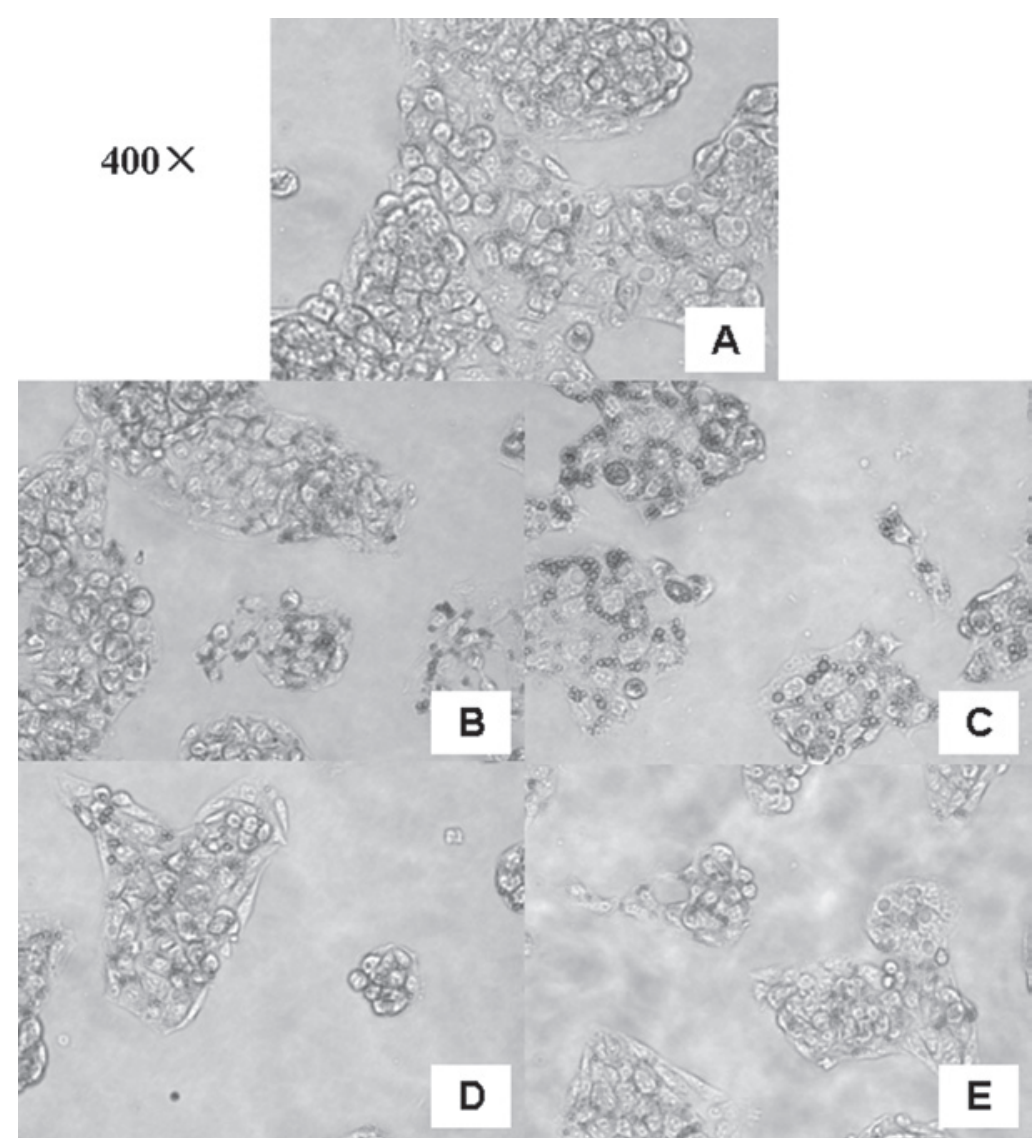

Figure 4. Oil red O staining results of HepG2 cells following the downregulation and upregulation of Nur77 expression. The quantification of TCHO levels was carried out in the same way for the five groups. (A) Blank group, (B) siRNA-NC and (C) siRNA-Nur77 were the downregulated groups. (D) pcDNA3.1mock and (E) pcDNA3.1-Nur77 were the upregulated groups. Following the downregulation of Nur77 expression, the lipid in the cells was increased in the siRNA-Nur77 group compared with that in the siRNA-NC and blank groups. On the contrary, following the upregulation of Nur77 expression, the lipid in the cells decreased in the pcDNA3.1-Nur77 group compared with that in the pcDNA3.1-mock and blank groups. These results are in agreement with the TCHO quantification results. TCHO, total cholesterol. 


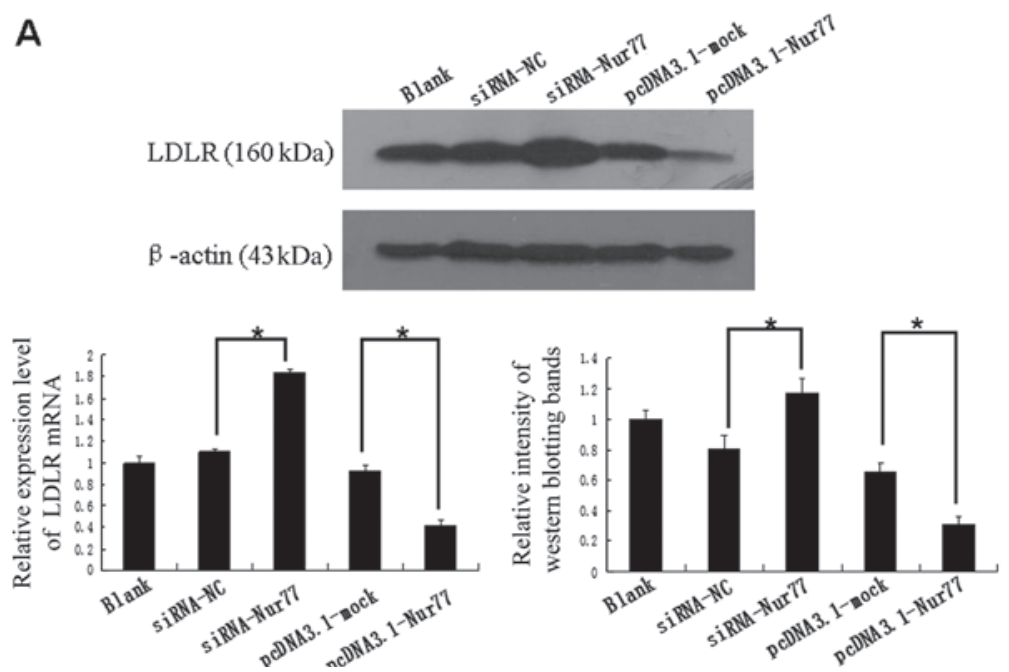

B
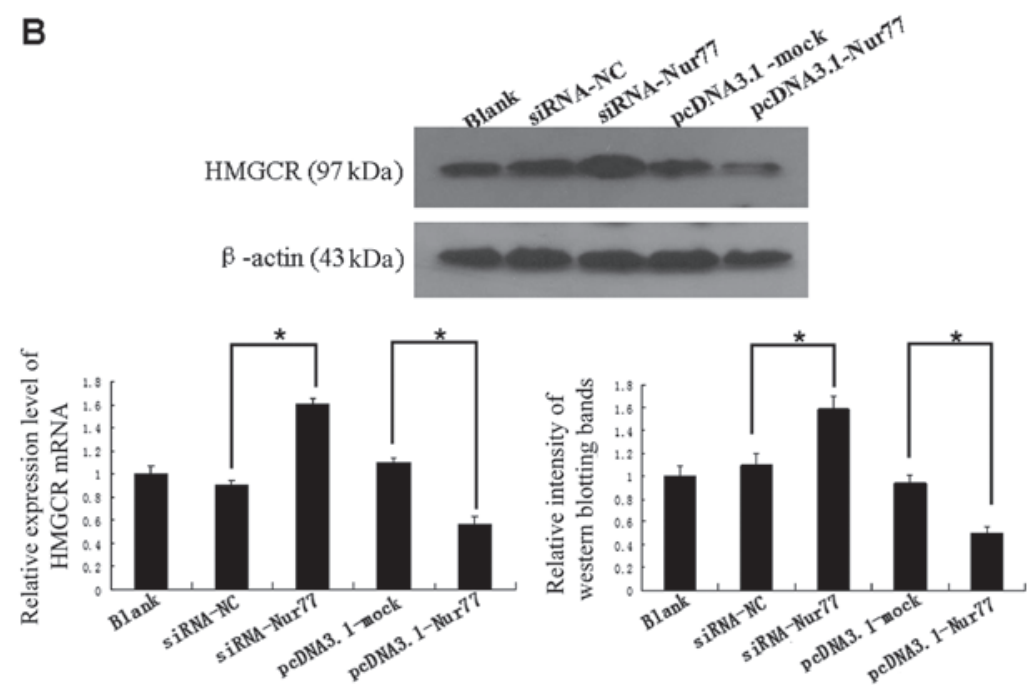

C

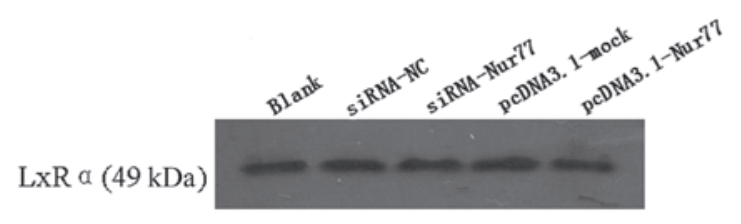

$\beta$-actin $(43 \mathrm{kDa})$
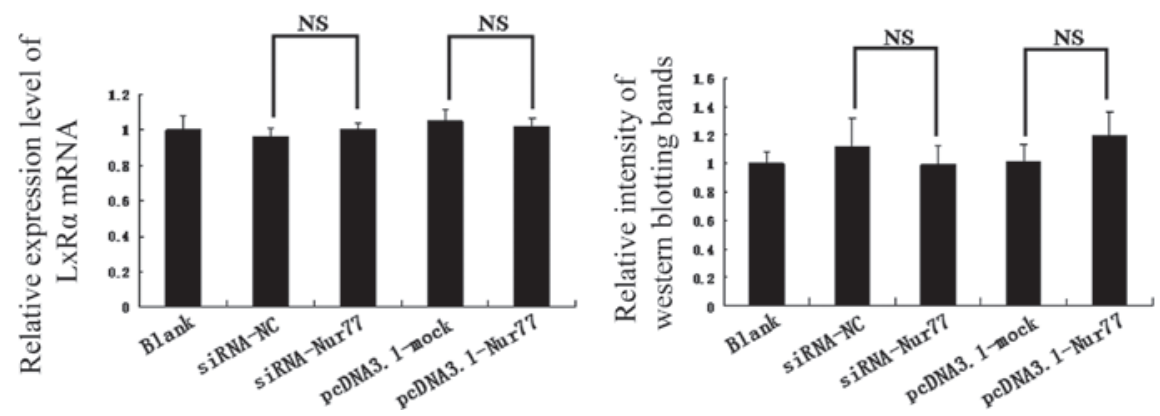

Figure 5. Changes in mRNA and protein levels of hepatic cholesterol metabolism genes following the downregulation and upregulation of Nur77 expression. (A) The relative expression level of LDLR mRNA increased following the downregulation of Nur77 expression and decreased following the upregulation of Nur77 expression. The differences between the siRNA-Nur77 group and the siRNA-NC group and those between the pcDNA3.1-Nur77 group and the pcDNA3.1-mock group were statistically significant ("P<0.01). Protein levels of LDLR among these groups experienced the same change. (B) As for HMGCR, the changes in mRNA and protein expression levels were similar to those of LDLR. (C) Relative expression levels of LXR $\alpha$ mRNA and protein did not change along with those of Nur77. There was no significant difference between the siRNA-Nur77 group and the siRNA-NC group, and also no significant difference between the pcDNA3.1-Nur77 group and the pcDNA3.1-mock group (NS, no significance; P>0.05). LDLR, low density lipoprotein receptor; HMGCR, HMGCoA reductase; $L X R \alpha$, liver X receptor $\alpha$. 
tion of plasma LLP. Therefore, the decrease in LDLR levels may contribute to the decrease in TCHO levels in the HepG2 cell in response to the overexpression of Nur77. HMGCR is the rate-limiting enzyme of hepatic cholesterol synthesis (22). It also experienced a decrease following the upregulation of Nur77 expression, and this may also play a role in the decrease of hepatic TCHO levels. Conversely, the expression level of HMGCR increased following the downregulation of Nur77 expression, and the hepatic TCHO level increased concomitantly. These results are in agreement with those from the animal study of Chao et al (15), which also demonstrated the correlation between HMGCR and Nur77 expression. LXR $\alpha$ is one of the most well-known regulators of hepatic cholesterol metabolism. It is the key factor in cholesterol catabolism and plays an important role in the synthesis and excretion of bile acid (23). However, to our disappointment, the Nur77-induced change in hepatic TCHO levels was not related to LXR $\alpha$.

To date, it has been confirmed that Nur77 is critical in the induction of apoptosis $(11,24)$. Therefore, there has been much interest in the possibility of a Nur77 agonist as a new potential anticancer drug. However, according to our findings, the hepatic cholesterol decreasing effect of Nur77 must be considered when evaluating this possibility.

In conclusion, we demonstrate that Nur77 plays a role in the regulation of hepatic cholesterol metabolism and reduces the cholesterol level in hepatocytes. The reason for this regulation may lie in the fact that Nur77 may reduce the hepatic expression of LDLR and HMGCR.

\section{Acknowledgements}

We thank Dr Tianyu Zhong and Dr Jie Bao for their technical assistance. This study was supported by the National Natural Science Foundation of China (No. 81071416).

\section{References}

1. Poli G, Sottero B, Gargiulo S, et al: Cholesterol oxidation products in the vascular remodeling due to atherosclerosis. Mol Aspects Med 30: 180-189, 2009.

2. Yoshida T, Kato K, Yokoi K, et al: Association of genetic variants with myocardial infarction in Japanese individuals with different lipid profiles. Int J Mol Med 25: 607-616, 2010.

3. Ooi K, Shiraki K, Sakurai Y, et al: Clinical significance of abnormal lipoprotein patterns in liver diseases. Int J Mol Med 15: $655-660,2005$.

4. Milbrandt J: Nerve growth factor induces a gene homologous to the glucocorticoid receptor gene. Neuron 1: 183-188, 1988.

5. Meinke G and Sigler PB: DNA-binding mechanism of the monomeric orphan nuclear receptor NGFI-B. Nat Struct Biol 6: 471-477, 1999.
6. Maxwell MA and Muscat GE: The NR4A subgroup: immediate early response genes with pleiotropic physiological roles. Nucl Recept Signal 4: e002, 2006.

7. Wang Z, Benoit G, Liu J, et al: Structure and function of Nurrl identifies a class of ligand-independent nuclear receptors. Nature 423: 555-560, 2003.

8. Giguère V: Orphan nuclear receptors: from gene to function. Endocr Rev 20: 689-725, 1999.

9. Yoon K, Lee SO, Cho SD, et al: Activation of nuclear TR3 (Nr4a1) by d diindolylmethane analog induces apoptosis and proapoptotic genes in pancreatic cancer cells and tumors. Carcinogenesis 32: 836-842, 2011.

10. Yang H, Zhan Q, Wan YJ, et al: Enrichment of Nur77 mediated by retinoic acid receptor $\beta$ leads to apoptosis of human hepatocellular carcinoma cells induced by fenretinide and histone deacetylase inhibitors. Hepatology 53: 865-874, 2011.

11. Wu Q, Liu S, Ye XF, et al: Dual roles of Nur77 in selective regulation of apoptosis and cell cycle by TPA and ATRA in gastric cancer cells. Carcinogenesis 23: 1583-1592, 2002.

12. Bonta PI, van Tiel CM, Vos M, et al: Nuclear receptors Nur77, Nurr1, and NOR-1 expressed in atherosclerotic lesion macrophages reduce lipid loading and inflammatory responses. Arterioscler Thromb Vasc Biol 26: 2288-2294, 2006.

13. Shao Q, Shen LH, Hu LH, et al: Nuclear receptor Nur77 suppresses inflammatory response dependent on COX-2 in macrophages induced by oxLDL. J Mol Cell Cardiol 49: 304-311, 2010.

14. Zhao Y, Liu Y and Zheng D: Alpha 1-antichymotrypsin/SerpinA3 is a novel target of orphan nuclear receptor Nur77. FEBS J 275: 1025-1038, 2008.

15. Chao LC, Wroblewski K, Zhang Z, et al: Insulin resistance and altered systemic glucose metabolism in mice lacking Nur77. Diabetes 58: 2788-2796, 2009.

16. Chao LC, Zhang Z, Pei L, et al: Nur77 coordinately regulates expression of genes linked to glucose metabolism in skeletal muscle. Mol Endocrinol 21: 2152-2163, 2007.

17. Pols TW, Ottenhoff R, Vos M, et al: Nur77 modulates hepatic lipid metabolism through suppression of SREBP1c activity. Biochem Biophys Res Commun 366: 910-916, 2008.

18. Gómez-Lechón MJ, Donato MT, Martínez-Romero A, et al: A human hepatocellular in vitro model to investigate steatosis. Chem Biol Interact 165: 106-116, 2007.

19. Song KH: Orphan nuclear receptor Nur77 participates in human apolipoprotein A5 gene expression. Biochem Biophys Res Commun 392: 63-66, 2010.

20. Navarro MA, Badimon L, Rodriguez C, et al: Trans-10,cis12-CLA dysregulate lipid and glucose metabolism and induce hepatic NR4A receptors. Front Biosci (Elite Ed) 2: 87-97, 2010.

21. Hussain MM, Strickland DK and Bakillah A: The mammalian low-density lipoprotein receptor family. Annu Rev Nutr 19: 141-172, 1999.

22. Trapani L, Segatto M, Simeoni V, et al: Short- and long-term regulation of 3-hydroxy 3-methylglutaryl coenzyme A reductase by a 4-methylcoumarin. Biochimie 93: 1165-1171, 2011.

23. Peet DJ, Turley SD, Ma W, et al: Cholesterol and bile acid metabolism are impaired in mice lacking the nuclear oxysterol receptor LXR $\alpha$. Cell 93: 693-704, 1998.

24. Li, H, Kolluri SK, Gu J, et al: Cytochrome c release and apoptosis induced by mitochondrial targeting of nuclear orphan receptor TR3. Science 289: 1159-1164, 2000. 